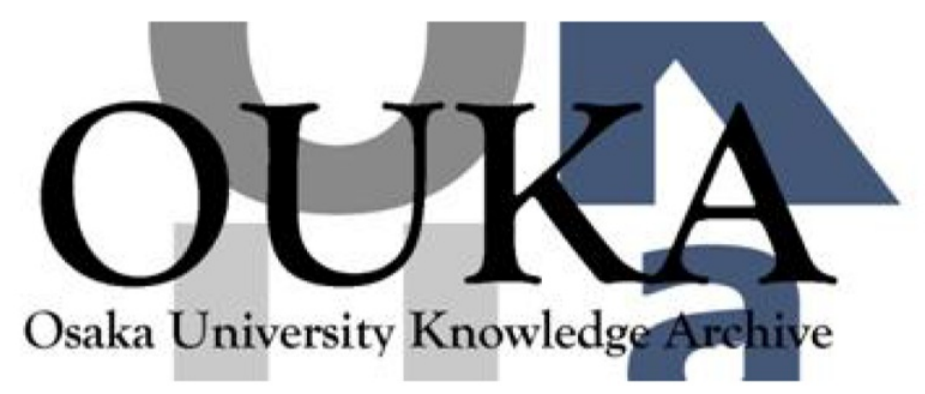

\begin{tabular}{|c|l|}
\hline Title & $\begin{array}{l}\text { Optical fractal synthesizer : concept and } \\
\text { experimental verification }\end{array}$ \\
\hline Author(s) & Tanida, Jun; Uemoto, Atsushi; Ichioka, Yoshiki \\
\hline Citation & Applied Optics. 32(5) p.653-p.658 \\
\hline Issue Date & $1993-02-10$ \\
\hline oaire:version & VoR \\
\hline URL & https://hdl. handle. net/11094/3331 \\
\hline rights & \\
\hline Note & \\
\hline
\end{tabular}

Osaka University Knowledge Archive : OUKA

https://ir. Library. osaka-u. ac. jp/

Osaka University 


\title{
Optical fractal synthesizer: concept and experimental verification
}

\author{
Jun Tanida, Atsushi Uemoto, and Yoshiki Ichioka
}

\begin{abstract}
An application of optical parallel processing in the generation of fractal images is presented. Iterated function systems [M. Barnsley, Fractals Everywhere (Academic, Boston, Mass., 1988), Chap. 3] are the basis of the operation, which can be easily implemented with optical techniques. An optical fractal synthesizer is considered to compute iterated function systems effectively with the advantages of optical processing in data continuity as well as parallelism. As an instance of the optical fractal synthesizer, an experimental system consisting of two optical subsystems for affine transformation and a TV-feedback line is constructed. Several experimental results verify the principle and show the processing capability of the optical fractal synthesizer.

Key words: Optical computing, fractal, iterated function system, affine transformation, image compression, feedback system.
\end{abstract}

\section{Introduction}

The massively parallel and high-speed nature of optical phenomena are expected to be useful in information processing. Much research has been stimulated by these attributes, and fruitful results have been obtained in the field of optical computing. ${ }^{1}$ Although the above two features are attractive and easy to apply to practical problems, there is another type of important feature in optics, i.e., continuity of the image. Most of the current research in image science is founded on discrete or sampled images because they are suitable for digital processing. The flexibility offered by the digital technique is powerful, but the discontinuity of the image causes undesired effects in some operations such as rotation, magnification, or reduction. Our purpose is to show how the third feature, continuity, can be used effectively in optical image processing.

The problem we engage in is generation of fractal images with an iterated function system (IFS). Fractals were introduced by Mandelbrot to treat a class of shapes, objects, or phenomena that cannot be treated with conventional mathematical methods. ${ }^{2}$ In mathematical terms a fractal is defined as a set

When this work was done, the authors were with the Department of Applied Physics, Osaka University, 2-1 Yamadaoka, Suita, Osaka 565, Japan. A. Uemoto is now with Seiko Instruments, Inc., 563 Takatsuka-shinden, Matsudo, Chiba 271, Japan.

Received 30 March 1992.

0003-6935/93/050653-06\$05.00/0.

() 1993 Optical Society of America. whose dimension in the Hausdorff-Besicovitch met$\mathrm{ric}^{2}$ is larger than the topological dimension. However, the term fractal is used here to refer to the shape projected upon an image plane to avoid unnecessary confusion.

According to research by Barnsley, a finite set of specific contractive mapping functions, or an IFS, can be used to generate a fractal. ${ }^{3}$ The contractive mapping function transforms any point in a fractal upon itself or upon another point in the fractal. If a point is transformed upon itself, the point is called a fixed point. Infinite repetition of the transformation provides a fractal as a set of the fixed points of the mapping, called an attractor. In the sequence an IFS determines the final status of the image, which means that parameters in the IFS can be used to generate a complex-shape fractal. Although the method is effective for the case in which a fractal can be described by a small number of parameters in the IFS, it is expected to be utilized for strong image compression. ${ }^{4}$

Unfortunately, the repeated transformations in the above sequence impose large amounts of computation. Usually, massive amounts of data must be used to process a large image. In addition, an IFS requires continuous image rotation and reduction, which is not suitable for discrete images. Therefore we present an optical fractal synthesizer (OFS) as an example of an optical computing system that uses the features of data continuity as well as parallelism in optics.

In the field of fractal generation by optical methods, 
Crutchfield ${ }^{5}$ and Häusler et al. ${ }^{6}$ presented excellent results by using TV-feedback systems. They demonstrated the capabilities of TV-feedback systems for generating chaos and for analyzing the systems. Although the system presented here is also based on a TV-feedback system, its processing principle, the IFS, is considered to be a different approach for fractal generation by optics.

A basic idea of the OFS is presented, and experimental results obtained by the preliminary system are shown. In Section 2 the theoretical basis of the IFS is explained briefly. In Section 3 a concept of the OFS is presented and an optical setup of its experimental system is described as an instance of the OFS. In Section 4, experimental results obtained by the experimental system are presented.

\section{Iterated Function System and Fractal Generation}

An IFS is defined as a function system that consists of a complete metric space $(\mathbf{X}, d)$ together with a finite set of contraction mappings $w_{n}: \mathbf{X} \rightarrow \mathbf{X}$ with respective contractivity factors $s_{n}$, for $n=1,2, \ldots, N$, where $\mathbf{X}$ is a space and $d$ is a real-valued function that is a metric for measuring distance. The contractivity factor $s$ of the IFS is obtained as the maximum in $s_{1}$, $s_{2}, \ldots, s_{N}$ and is $0 \leq s<1$ for contraction mapping. ${ }^{3}$

Barnsley showed that the transformation $W$ : $\mathscr{H}(\mathbf{X}) \rightarrow \mathscr{H}(\mathbf{X})$, defined by $W(B)=W_{1}(B) \cup W_{2}(B) \cup$ $\cdots \cup W_{N}(B)$ for all $B \in \mathscr{H}(\mathbf{X})$, is a contraction mapping on the complete metric space $[\mathscr{H}(\mathbf{X}), h(d)]$ with contractivity factor $s .^{3} \mathscr{H}(\mathbf{X})$ denotes the space whose points are the compact subsets of $\mathbf{X}$ other than the empty set. $h(d)$ denotes that $d$ is the underlying metric for the Hausdorff metric $h .^{3}[\mathscr{H}(\mathbf{X}), h(d)]$ is the space of fractals, and any subset of $[\mathscr{H}(\mathbf{X}), h(d)]$ is a fractal.

The unique fixed point of the above transformation, $A \in \mathscr{H}(\mathbf{X})$, obeys $A=W(A)=W_{1}(A) \cup W_{2}(A) \cup$ $\cdots \cup W_{N}(A)$ and is given by $\lim _{n \rightarrow \infty} W^{0 n}(B)$ for any $B \in \mathscr{H}(\mathbf{X})$, where $W^{0 n}$ indicates $n$ times of sequential application of transformation $W$ on a point. The fixed point is called the attractor of the IFS.

The practical meaning of the above theory is that infinite transformation of an IFS for an arbitrary two-dimensional image will produce a fractal determined by the IFS. The process is shown in Fig. 1. In the figure the process of sequential application of a transformation, $W=W_{1} \cup W_{2}$, is shown. The arrows labeled by $W_{1}$ and $W_{2}$ indicate correspondence between the original and the transformed images of the individual transformation. In this process the initial image does not affect the result, so that parameters in the IFS are sufficient to describe the final fractal. Since the parameters in an IFS are much less than the total information of the final image, image compression of a fractal can be achieved by finding the IFS for a given fractal. ${ }^{3,7}$ A compression ratio of $10^{4}: 1$ is reported with this technique. ${ }^{4}$

There are two algorithms for computing, or generating, fractals with IFS's. ${ }^{3}$ One is the deterministic algorithm, and the other is the random-iteration
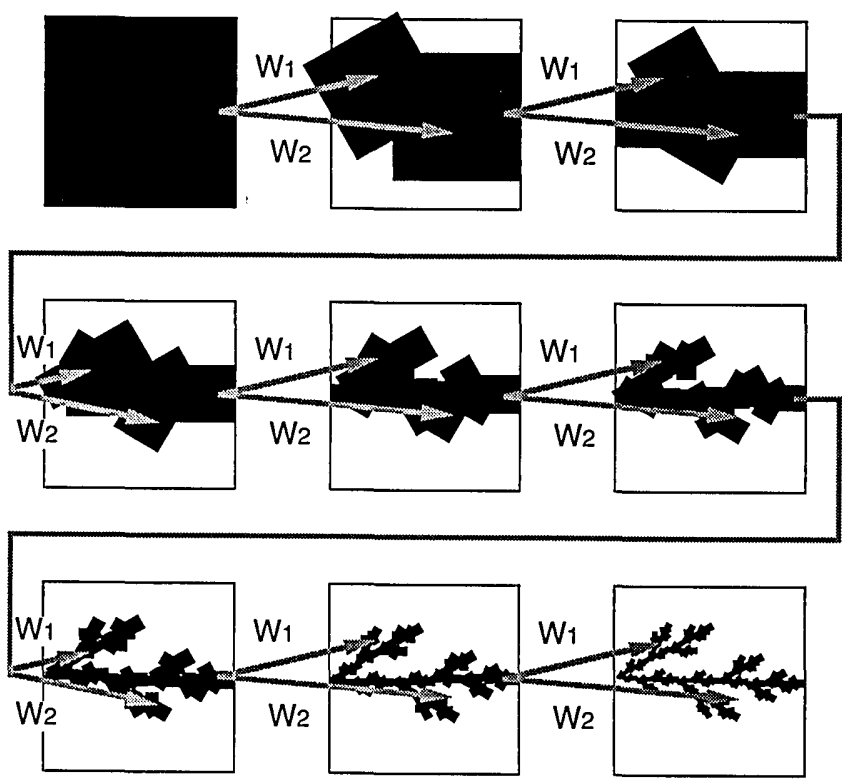

Fig. 1. Fractal generation with an IFS.

algorithm. In the deterministic algorithm, all points in an image are transformed point by point with one of the transformations, e.g., $W_{1}$, in the IFS. Then, with the transformation other than the one already used, e.g., $W_{2}$, the same process is repeated. For all results of the process above, the logical sum is operated, which provides the result of one iteration of the transformation $W=W_{1} \cup W_{2} \cup \cdots W_{N}$. According to the IFS scheme, the procedure is repeated by a sufficient number. On the other hand, the random-iteration algorithm introduces probability theory to reduce the total amount of computation. Although the random-iteration algorithm is suitable for current sequential computers and is a popular method, the deterministic algorithm is attractive for parallel computing systems, including neural networks. ${ }^{8}$ Therefore we adopt the deterministic algorithm as the computing algorithm of the OFS.

\section{Optical Fractal Synthesizer}

The OFS is an optical computing system for generating fractals from IFS's with the deterministic algo-

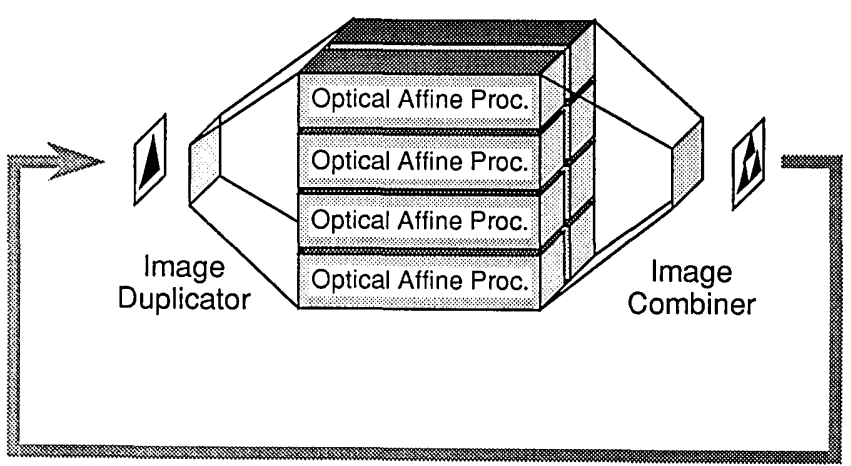

Optical Feedback Line

Fig. 2. Conceptual diagram of the OFS. 
Table 1. IFS Code Used for the Fractal Generated in the Clocked Mode ${ }^{a}$

\begin{tabular}{rrrrrrc}
\hline$i$ & $a$ & \multicolumn{1}{c}{$b$} & \multicolumn{1}{c}{$c$} & \multicolumn{1}{c}{$d$} & $e$ & $f$ \\
\hline 1 & 0.49 & -0.26 & -0.26 & -0.57 & 35.9 & 210.4 \\
2 & 0.51 & 0.29 & 0.26 & -0.58 & 87.2 & 144.1 \\
\hline
\end{tabular}

${ }^{a}$ See Fig. 4.

rithm. Figure 2 is a conceptual diagram of the OFS. An input image, usually a plain bright image, is duplicated and input to optical affine transform processors that transform the image by rotation, reduction, or other operations. The transformed images are combined into an image and fed back for the next iteration. After a sufficient number of iterations, a fractal is obtained whose shape is determined by the characteristics of the optical affine transform processors. Since the required operations in the system, i.e., duplication, rotation, reduction, and combination, are easily implemented by optical techniques, the OFS is expected to reveal the potential power of optical computing techniques.

To verify the concept of the OFS, we constructed an experimental system. For simplicity, contractive affine mapping is assumed for IFS's implemented on the system. Namely, the following transform is used for mapping:

$$
\begin{aligned}
W_{i}(\mathbf{X}) & =W_{i}\left[\begin{array}{l}
x \\
y
\end{array}\right]=\left[\begin{array}{ll}
a_{i} & b_{i} \\
c_{i} & d_{i}
\end{array}\right]\left[\begin{array}{l}
x \\
y
\end{array}\right]+\left[\begin{array}{l}
e_{i} \\
f_{i}
\end{array}\right], \\
& i=1,2, \ldots, N, \\
0 & <\left|a_{i} d_{i}-b_{i} c_{i}\right|<1,
\end{aligned}
$$

where $(x, y)$ indicates the location on an image plane. In Eq. (1) the $2 \times 2$ matrix provides rotation and reduction, and the $2 \times 1$ vector gives translation. By the $2 \times 2$ matrix, the individual axes $X$ and $Y$ of the rectangular Cartesian coordinates are independently magnified and rotated. The magnification ratio is $\left(a_{i}^{2}+b_{i}^{2}\right)^{1 / 2}$ for the axis $X$ and $\left(c_{i}^{2}+d_{i}^{2}\right)^{1 / 2}$ for the axis $Y$; the rotation angle is obtained by $\arctan \left(b_{i} / a_{i}\right)$ for

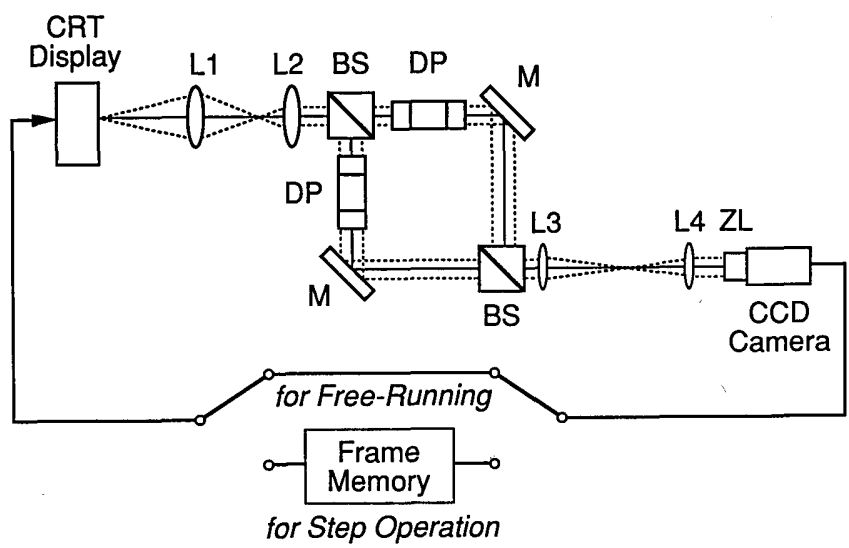

Fig. 3. Optical setup of the experimental OFS: BS's, beam splitters; DP's, dove prisms; ZL, zoom lens, L1-L4, lenses; M's, mirrors. the axis $X$ and $\arctan \left(-c_{i} / d_{i}\right)$ for the axis $Y$. The parameters used in Eq. (1) are expressed as values in Table 1. The data in the table are called an IFS code. $^{3}$ Inequality (2) is the condition for contractive mapping.

In addition, to simplify the optical setup, we also assume the following relations:

$$
\begin{aligned}
a_{i} & =-d_{i}, \\
b_{i} & =c_{i}, \\
N & =2, \\
\left|a_{1} d_{1}-b_{1} c_{1}\right| & =\left|a_{2} d_{2}-b_{2} c_{2}\right| .
\end{aligned}
$$

Equations (3) and (4) guarantee homogeneous reduction on the image plane, and Eq. (6) states that the reduction ratios of the two mappings are the same. These conditions are useful for simplifying the optical
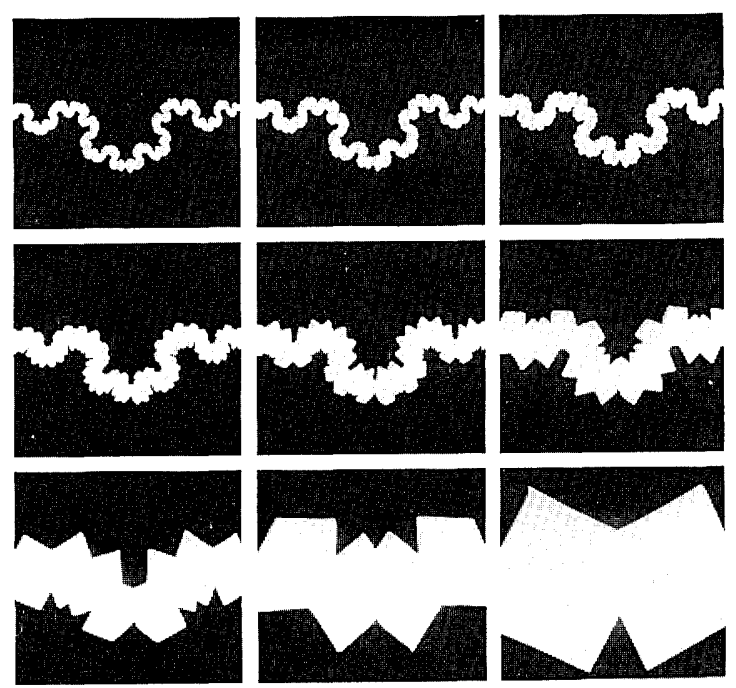

(a)
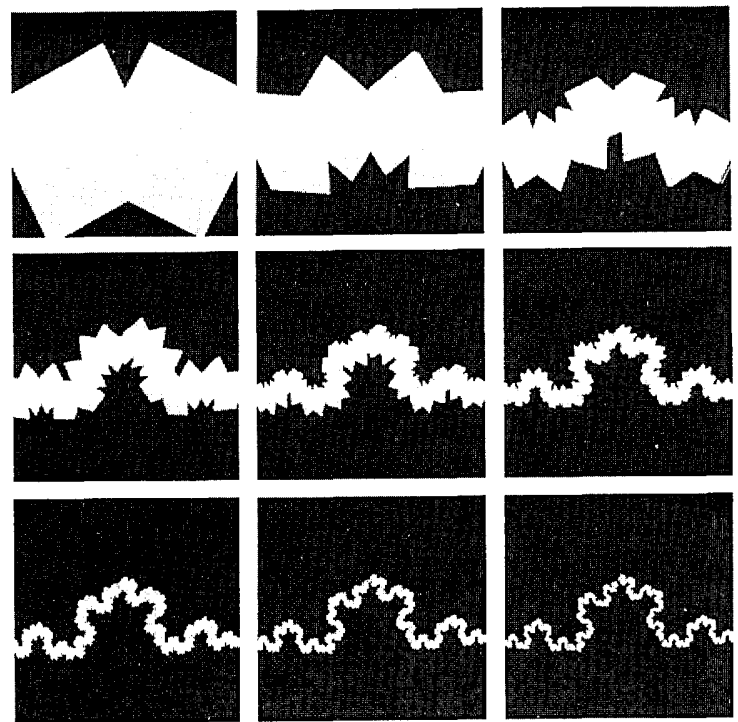

(b)

Fig. 4. Comparison between the fractals calculated (a) by the experimental OFS and (b) by the Sun SPARCStation 2. 


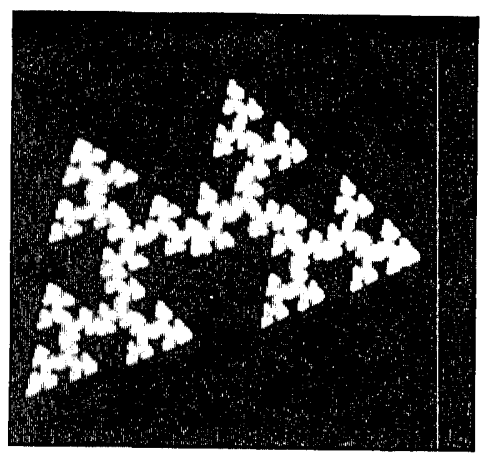

(a)

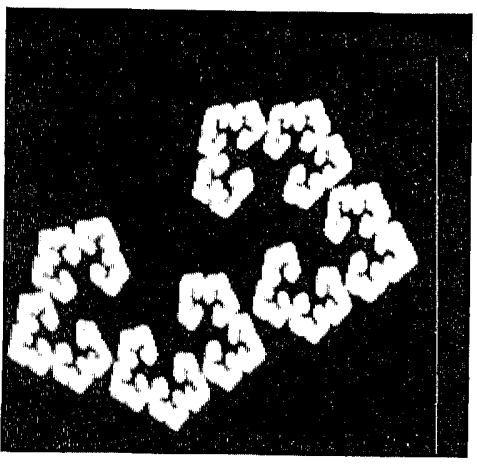

(d)

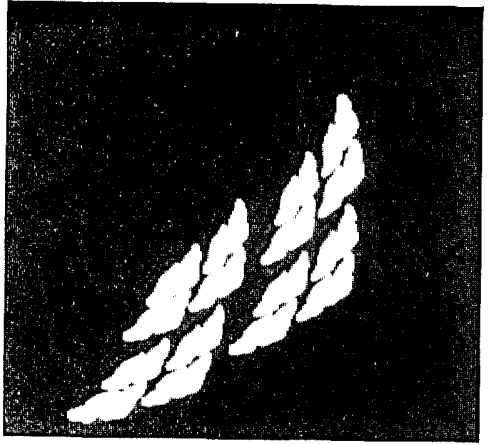

(b)

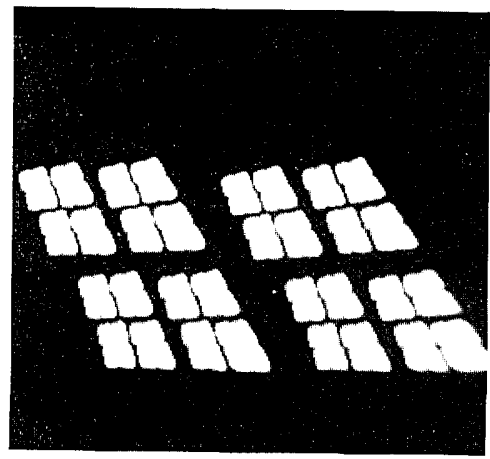

(e)

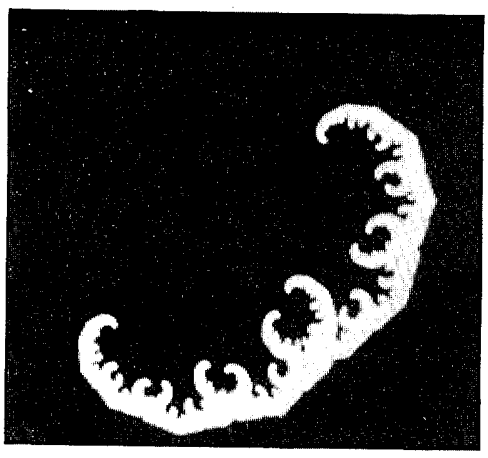

(c)

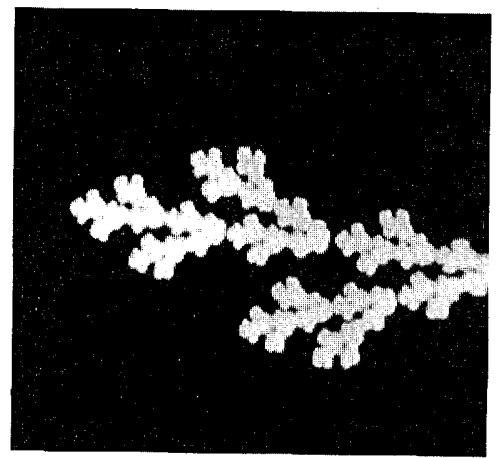

(f)

Fig. 5. Fractals obtained by the experimental OFS with the free-running operational mode.

setup. Equation (5) is simply the number of optical affine transform processors. Although the flexibility of the simplified system is greatly reduced, it is sufficient to verify the basic principle of the OFS. Even with two mapping IFS's, various fractals can be generated, as shown in Section 4.

Figure 3 shows the optical setup of the constructed system. In the system a CRT display and a CCD camera are used to feed back intermediate results. ${ }^{5,6,9}$ A frame memory $(256 \times 240$ pixels with 8 bits/pixel $)$ is inserted to increase the flexibility of system operation. To emphasize the merit of data continuity, we prefer an all-optical feedback system with samplingfree spatial light modulators. However, we need to consider that the availability of devices and the flexibility of the feedback operation are more important than pure parallelism at this time. As an advantage of the system, two operational modes, i.e., clocked and free-running feedbacks, can be selected by control of the frame memory in the feedback loop.

Affine transforms in Eq. (1) are implemented by dove prisms, mirrors, and a zoom lens in front of the CCD camera. The rotation angle of the dove prisms and the magnification ratio of the lens system determine the parameters $a_{i}, b_{i}, c_{i}$, and $d_{i}(i=1,2)$ in Eq. (1). The tilt angle of the mirrors in each branch provides a translation described by $e_{i}$ and $f_{i}(i=1,2)$ in Eq. (1). In the experimental system these parameters are simply adjusted.

\section{Experimental Results}

To check the correct operation of the system, we generated the same fractal on the experimental OFS and on a Sun SPARCStation 2. Figure 4 shows the results obtained. In this case the OFS is operated in the clocked mode, in which all operations of the system obey discrete clocks. The IFS code used is indicated in Table 1; the code is calculated from the

Table 2. IFS Codes Used for the Fractals Generated in the Free-Running Iteration Mode ${ }^{a}$

\begin{tabular}{crrrrrr}
\hline$i$ & $a$ & $b$ & $c$ & \multicolumn{1}{c}{$d$} & \multicolumn{1}{c}{$e$} & \multicolumn{1}{c}{$f$} \\
\hline$(a)-1$ & 0.57 & 0.25 & 0.21 & -0.65 & 61.2 & 171.2 \\
$(a)-2$ & -0.13 & -0.66 & -0.60 & 0.11 & 161.3 & 193.4 \\
\hline$(b)-1$ & 0.31 & 0.58 & 0.51 & -0.36 & 35.7 & 114.2 \\
$(b)-2$ & 0.16 & 0.64 & 0.60 & -0.18 & -24.0 & 125.3 \\
\hline$(c)-1$ & 0.31 & 0.59 & 0.51 & -0.36 & 51.0 & 101.2 \\
$(c)-2$ & -0.32 & 0.56 & 0.51 & 0.37 & 74.2 & 56.8 \\
\hline$(d)-1$ & 0.57 & 0.24 & 0.21 & -0.65 & 61.5 & 171.2 \\
$(d)-2$ & -0.56 & 0.17 & 0.17 & 0.66 & 117.6 & 56.3 \\
\hline$(e)-1$ & 0.31 & 0.60 & 0.52 & -0.36 & 56.5 & 121.1 \\
$(e)-2$ & 0.29 & 0.58 & 0.54 & -0.33 & -46.6 & 115.3 \\
\hline$(f)-1$ & 0.61 & -0.05 & -0.05 & -0.70 & 95.2 & 247.1 \\
$(f)-2$ & 0.56 & 0.19 & 0.17 & -0.66 & -6.8 & 173.5 \\
\hline
\end{tabular}

${ }^{a}$ Seo Fig. 5. 

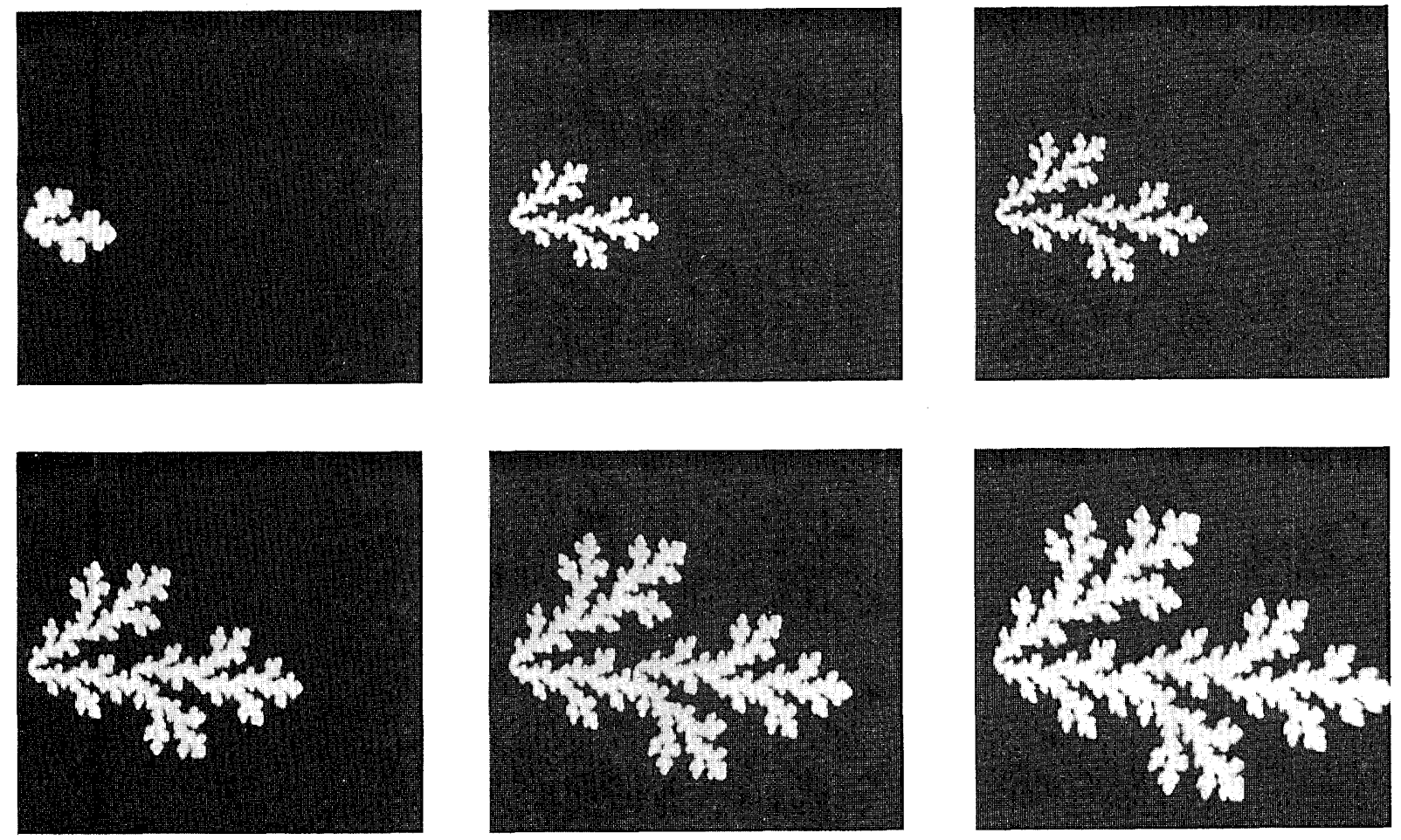

Fig. 6. Series of fractals obtained by continuous change of the system parameters.

transformation of three individual points on the optical setup. Note that these codes depend on the coordinate system of the frame memory, with $0 \leq$ $x \leq 255$ and $0 \leq y \leq 239$. Figure 4 shows that nearly the same fractal is obtained by the two systems. In this experiment the OFS executes one iteration within $0.1 \mathrm{~s}$, while the SPARCStation 2 requires $6 \mathrm{~s}$ to complete the same operation. The speed of the OFS is limited by the CRT and CCD devices.

To demonstrate the capability of generating a variety of fractals with the experimental OFS, we generated several fractals by using the free-running iteration mode. Figure 5 shows the results. In this experiment, by observing the images on the CRT, we adjusted the rotation angles, the magnification ratio, and the shift amounts to find interestingly shaped fractals. Thus the IFS codes for the fractals are calculated from the transformation of three individual points on the optical setup after the setting. The obtained IFS codes are listed in Table 2. As seen from Tables 1 and 2, Eqs. (3) and (4) are not satisfied by the observed IFS codes. Since Eqs. (3) and (4) are ideal conditions for the simplified system, this fact shows that misalignment, or aberration, remains in the experimental OFS. However, it also indicates that if we can control such aberration, ${ }^{10}$ more flexibility can be attained for the OFS.

We also observed that a fractal can be generated from another fractal on the OFS. Usually, a fractal is generated from an initial image, as shown in Fig. 1. However, if a fractal is already generated and remains on the CRT of the OFS, slightly deformed fractals can be generated directly from the fractal. Figure 6 shows an example of a series of fractals obtained by changing the system parameters in the free-running iteration mode. Since this process does not require a return to the initial stage, the processing time is greatly reduced.

\section{Discussion}

On the OFS, infinite iteration in the IFS scheme is not necessarily required because of the physical resolution of the optical system and devices. In practice, a finite number of iterations is sufficient for generating a fractal under limited resolution. However, the convergence of the iteration is affected by various factors such as the geometrical unstability of the TV-feedback system, the IFS code itself, the brightness of the image, and the density of bright points on the image. Of these factors the unstability of the TV-feedback system is the most detrimental and cannot be removed. As a result, in some cases a fractal is obtained; in other cases, no image is left after several iterations. In the clocked operational mode, such a disadvantage can be avoided by limiting the iteration number. Although the iteration number must be determined experimentally, this technique is useful for obtaining fine structure.

There is another flexibility obtained by configuring IFS codes with the experimental OFS. As is well known, an odd number of reflections provides a mirror image. In the setup of Fig. 3 an image displayed on the CRT reaches the CCD camera after three reflections (two by beam splitters and one by a dove prism). Thus the detected image is a mirrored one. If the CCD camera is moved to observe the other branch after the second beam splitter, a normal image is obtained on the CCD camera. In this case, 
Eqs. (3) and (4) are changed as follows:

$$
\begin{aligned}
& a_{i}=d_{i}, \\
& b_{i}=-c_{i} .
\end{aligned}
$$

As discussed in Section 3, the experimental system is a greatly simplified one. Although a variety of fractals can be generated on the system, an advanced system is required to explore the advantages of the OFS. There are two methods to reach this goal: constructing a pure optical system with sampling-free spatial light modulators and increasing the number of optical affine transform processors for individual contractive mappings in IFS's. For the former, a spatial light modulator with high spatial resolving power must be developed, and for the latter, a more sophisticated configuration must be developed for the optical setup. Such a pure optical system makes good use of the advantages both of optical processing in data continuity as well as of parallelism.

The OFS has a function for decompression in the image-compression scheme. ${ }^{3}$ Thus an effective method for image compression with optical computing techniques is strongly desired. This subject of research is attractive and promising because the features in optical processing can be fully utilized. These are the problems that we should attempt to solve next.

\section{References}

1. D. G. Feitelson, Optical Computing (MIT, Cambridge, Mass., 1988), Chaps. 3, 4, and 6-9.

2. B. B. Mandelbrot, The Fractal Geometry of Nature (Freeman, San Francisco, Calif., 1983), Chap. 3.

3. M. Barnsley, Fractals Everywhere (Academic, Boston, Mass., 1988), Chap. 3.

4. M. F. Barnsley and A. D. Sloan, "A better way to compress images," Byte 13, 215-223 (1988).

5. J. P. Crutchfield, "Space-time dynamics in video feedback," Physica (Utrecht) 10D, 229-245 (1984).

6. G. Häusler, G. Seckmeyer, and T. Weiss, "Chaos and cooperation in nonlinear pictorial feedback systems. 1: Experiments," Appl. Opt. 25, 4656-4663 (1986).

7. S. Kocsis, "Digital compression and iterated function systems," in Applications of Digital Image Processing XII, A. G. Tescher, ed., Proc. Soc. Photo-Opt. Instrum. Eng. 1153, 19-27 (1989).

8. J. Stark, "Iterated function systems as neural networks," Neural Networks 4, 679-690 (1991).

9. G. Ferrano and G. Häusler, "TV optical feedback systems," Opt. Eng. 19, 442-451 (1980).

10. A. W. Lohmann and N. Streibl, "Map transformations by optical anamorphic processing,” Appl. Opt. 22, 780-783 (1983). 